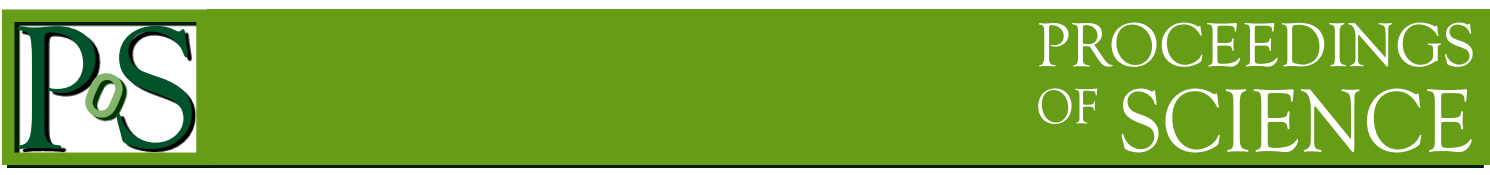

\title{
"ALTIROC0, a 20 pico-second time resolution ASIC for the ATLAS High Granularity Timing Detector (HGTD)"
}

\author{
C. de La Taille ${ }^{1}$ \\ OMEGA/CNRS/Ecole Polytechnique \\ Ecole Polytechnique - Drahi-X Novation Center \\ Avenue Coriolis \\ 91128 PALAISEAU CEDEX \\ FRANCE \\ E-mail: tailleein2p3.fr
}

\section{Other Authors}

\section{S. Callier, S. Conforti, P. Dinaucourt, G. Martin-Chassard, N. Seguin-Moreau} OMEGA/CNRS/Ecole Polytechnique

E-mail: nsmoreaulin2p3. fr

\section{Agapopoulou, N. Makovec, L. Serin, S. Simion}

LAL/CNRS/Université Paris Sud

Laboratoire de l'Accélérateur Linéaire (LAL)

Université Paris-Sud

91405 ORSAY CEDEX

FRANCE

E-mail: serinllal.in2p3.fr

ALTIROC0 is an 8-channel ASIC prototype designed to readout $1 \times 1$ or $2 \times 2 \mathrm{~mm}^{2} 50 \mu \mathrm{m}$ thick Low Gain Avalanche Diodes (LGAD) of the ATLAS High Granularity Timing Detector (HGTD). The targeted combined time resolution of the sensor and the readout electronics is $30 \mathrm{ps}$ for one MIP. Each analog channel of the ASIC must exhibit an extremely low jitter to ensure this challenging time resolution, while keeping a low power consumption of $2 \mathrm{~mW} /$ channel. A "Time Over Threshold" and a "Constant Fraction Discriminator" architecture are integrated to correct for the time walk.

Test bench measurements performed on the ASIC received in April 2017 are presented.

Topical Workshop on Electronics for Particle Physics

11 - 14 September 2017

Santa Cruz, California

\section{${ }^{1}$ Speaker}




\section{Introduction}

The expected increase of the pile-up at the high luminosity phase of the LHC due to the 200 interactions per bunch crossing will have a severe impact on the physics [1]. A High Granularity Timing Detector (HGTD) is proposed in front of the ATLAS Liquid Argon End-Cap calorimeters for pile-up mitigation especially for jets and electron isolation. Four layers of very thin Low Gain Avalanche Diodes (LGAD), with a pad size of $1.3 \times 1.3 \mathrm{~mm}^{2}$, are foreseen. This detector is expected to provide a precise timing information for charged particles with a time resolution of about 30 pico-seconds for one MIP, combining the 4 layers after irradiation. It will also be used to provide a luminosity measurement at $40 \mathrm{MHz}$ by counting the number of hits.

The sensors will be read-out by a dedicated front-end ASIC called ALTIROC (ATLAS LGAD Timing Integrated Read-Out Chip), that will be bump-bonded on the sensors. A first prototype comprising only the analog part of this ASIC has been designed in CMOS $130 \mathrm{~nm}$ technology to cope with the high radiation and time resolution requirements, while keeping a power dissipation lower than $2 \mathrm{~mW} /$ pixel for a complete readout channel.

\section{ALTIROC0 architecture}

\subsection{ASIC requirements}

The requirements of the final ASIC (ALTIROC) are listed in Table 1.

\begin{tabular}{|l|l|}
\hline Pad size & $1.3 \times 1.3 \mathrm{~mm}^{2}$ \\
\hline Detector capacitance & $3.4 \mathrm{pF}$ \\
\hline TID and neutron fluence & $\begin{array}{l}\text { Inner region: } 4.5 \mathrm{MGy}, 4.5 \times 10^{15} \mathrm{n} / \mathrm{cm}^{2} \\
\text { Outer region: } 2.1 \mathrm{MGy}, 4.0 \times 10^{15} \mathrm{n} / \mathrm{cm}^{2}\end{array}$ \\
\hline Number of channels/ASIC & 225 \\
\hline Minimum threshold & $\mathbf{5} \mathrm{fC}$ (=0.5 MIP at LGAD gain $=20)$ \\
\hline Dynamic range & $20 \mathrm{MIPs}$ \\
\hline Preamplifier-Discri jitter at Gain=20 & $<20 \mathrm{ps}$ \\
\hline Time Walk contribution & $<10 \mathrm{ps}$ \\
\hline TDC bin & $20 \mathrm{ps}$ (TOA) and $40 \mathrm{ps}$ (TOT) \\
\hline TDC range & $2.5 \mathrm{~ns}(\mathrm{TOA})$ and $9 \mathrm{~ns}$ (TOT) \\
\hline Number of bits/hit & 7 for TOA and 9 for TOT \\
\hline Luminosity counters per ASIC & $7 \mathrm{bits}(\mathrm{sum})+5 \mathrm{bits}$ (outside window) \\
\hline Total power per area (ASIC) & $<200 \mathrm{~mW} / \mathrm{cm}^{2}(<800 \mathrm{~mW})$ \\
\hline elink driver bandwidth & $320 \mathrm{Mb} / \mathrm{s}, 640 \mathrm{Mb} / \mathrm{s}$ and $1.28 \mathrm{~Gb} / \mathrm{s}$ \\
\hline
\end{tabular}

Table 1: ALTIROC final ASIC requirements

In particular, the ASIC must withstand high radiation levels with a maximum TID of 4.5 MGy at small radius and neutron fluence of $4.5 \times 10^{15} \mathrm{n} / \mathrm{cm}^{2}$. The inner part of the detector, from $\mathrm{r}=120 \mathrm{~mm}$ to $300 \mathrm{~mm}$, is expected to be changed at half lifetime of the HL-LHC. Each channel will read out $1.3 \times 1.3 \mathrm{~mm}^{2}$ sensor pads, corresponding to a detector capacitance of 3.4 $\mathrm{pF}$. The targeted time resolution is $30 \mathrm{ps} r m s$ for one MIP with 4 layers after irradiation. To 
achieve such a performance, the jitter must be better than $20 \mathrm{ps}$ in order to be smaller than the dispersion due to the Landau fluctuation in the energy deposit, which limits the time resolution to $25 \mathrm{ps}$. As for the time walk contribution, it must be corrected with an accuracy leading to a residual contribution better than $10 \mathrm{ps} r m s$ for signals ranging from 1 MIP to 10-20 MIPs, which corresponds to 10 to $100-200 \mathrm{fC}$ with an LGAD gain equal to 20 . The power dissipation of a complete pixel readout must be limited to $2 \mathrm{~mW}$, split in $1 \mathrm{~mW}$ for the analog part and $1 \mathrm{~mW}$ for the Time to Digital Convertors (TDC) and digital processing.

\subsection{Analog front end of ALTIROCo}

ALTIROC0 is a first ASIC prototype designed in CMOS $130 \mathrm{~nm}$ that integrates only the analog front end, made of a broadband preamplifier followed by a discriminator (Figure 1).

The jitter due to electronics noise is given by the rise time divided by the signal to noise ratio. To obtain high speed, the input preamplifier is built around a common source configuration. It was optimized for a $2 \mathrm{pF}$ detector capacitance corresponding to an earlier $1 \mathrm{x} 1$ $\mathrm{mm}^{2}$ sensor prototype. Its bandwidth is tunable between $300 \mathrm{MHz}$ and $500 \mathrm{MHz}$ using a variable pole capacitance and its noise has been minimized to $1.2 \mathrm{nV} / \sqrt{\mathrm{Hz}}$ at $200 \mathrm{uA}$ drain current to ensure a large Signal over Noise ratio and therefore a jitter smaller than $20 \mathrm{ps} r m s$.

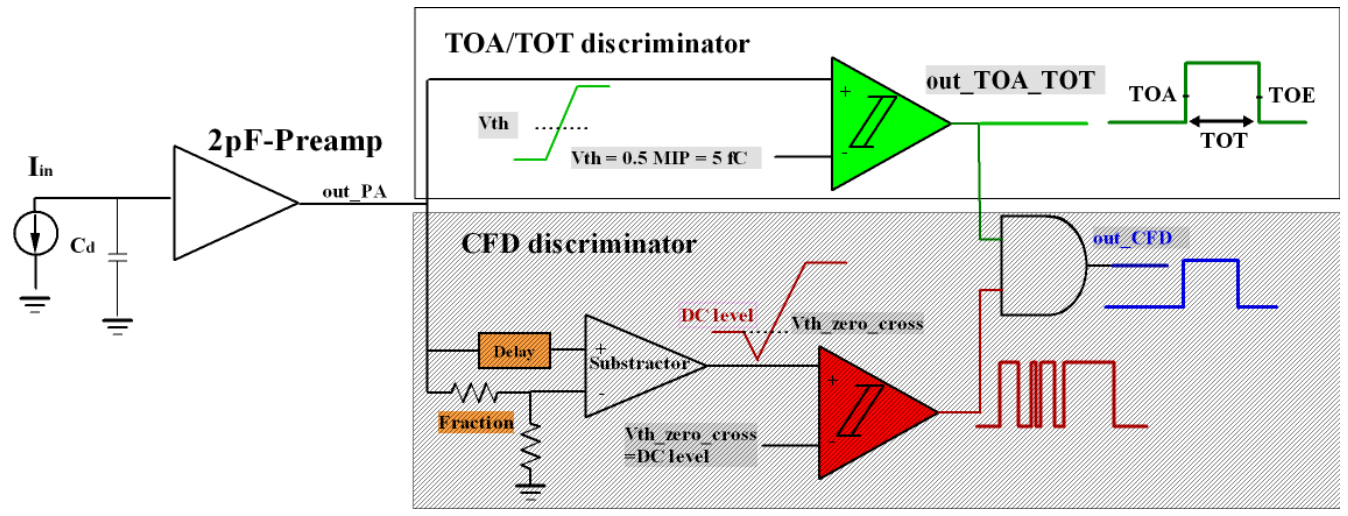

Figure 1: ALTIROCO Front End synoptic

Two time-walk correction architectures, both built around leading edge discriminators, have been integrated to correct for the Time Walk of approximately $1 \mathrm{~ns}$ :

- A Time Over Threshold (TOT) architecture, that enables offline time walk correction using the width of the discriminator (TOT), which is proportional to the amplitude

- A Constant Fraction Discriminator (CFD), which is in theory insensitive to the amplitude variations if the pulse shape remains constant and so, allowing online time walk correction.

The chip area is $3.4 \mathrm{~mm} \times 3.4 \mathrm{~mm}$ to enable its bump-bonding to an array of $2 \times 2$ channels with four $1 \times 1 \mathrm{~mm}^{2}$ LGAD sensor pixels. 


\section{ALTIROC0 measurements}

\subsection{ALTIROC0 prototype}

This first ASIC prototype integrates four channels to readout four $1 \mathrm{x} 1 \mathrm{~mm}^{2}$ LGAD pixels. The dies were received in April 2017. A testboard has been designed to allow measurements of either an ASIC alone, wire bonded on the printed circuit board, or a flip chip made of an ASIC bump-bonded to a 4 pixel sensor.

\subsection{ALTIROC0 jitter measurements}

Only the TOT architecture was tested so far (Figure 1). The rising edge of the discriminator corresponds to the Time Of Arrival (TOA). Its position and its jitter were measured for input charges ranging from $8 \mathrm{fC}$ up to $160 \mathrm{fC}$ with a threshold set at $5 \mathrm{fC}$. Various capacitors $(\mathrm{Cd})$ were soldered on the test board to simulate the sensor. A parasitic capacitance, estimated to $1.3 \mathrm{pF}$, should actually be added to the soldered detector capacitance, which corresponds to the capacitance of the preamp input protection diode $(\approx 0.7 \mathrm{pF})$ and to the capacitance of the printed circuit board line $(\approx 0.6 \mathrm{pF})$ that connects the input preamp to the detector capacitor foot print. Figure 2 shows the results with a $2 \mathrm{pF}$ detector capacitor as it corresponds to the expected capacitance of a $1 \times 1 \mathrm{~mm}^{2}$ LGAD pixel. The rise time of the preamplifier has been estimated by measuring the time walk. To do so, the position of the TOA has been measured for input charges going from $8 \mathrm{fC}$ up to $160 \mathrm{fC}$. The time walk amounts to 1 ns, corresponding to a preamp bandwidth of $450 \mathrm{MHz}$ that is in good agreement with post layout simulations.
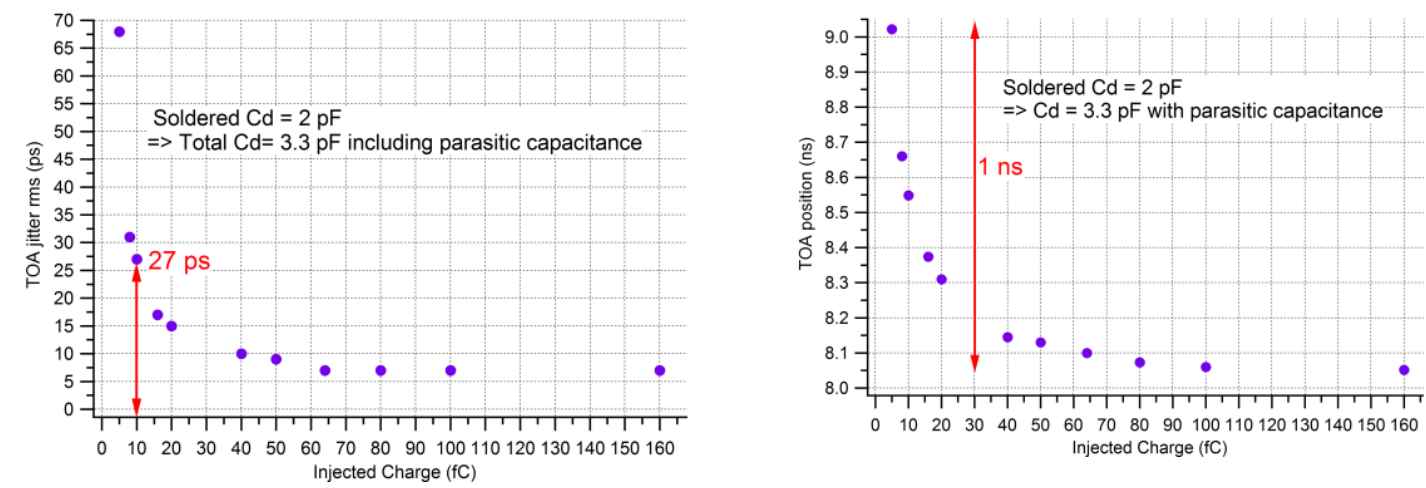

Figure 2: TOA jitter rms and position vs the injected charge with $C d=2 \mathrm{pF}$ (actual $C d=3.3 \mathrm{pF}$ )

Table 2 summarizes the jitter performance obtained for an injected charge of $10 \mathrm{fC}$ (corresponding to $1 \mathrm{MIP}$ ) and for various detector capacitors. The threshold was set at $5 \mathrm{fC}$. As expected, the measured jitter scales almost linearly with the capacitance. In particular, the jitter measured when a $2 \mathrm{pF}$ capacitor is soldered on the test board is $27 \mathrm{ps}$, which is slightly larger than the requirement, but this could certainly be improved by increasing the current in the preamp to reduce the noise. 


\begin{tabular}{|l|c|c|}
\hline Cd & Actual Cd & Jitter @ 10 fC \\
\hline $\mathbf{0} \mathbf{p F}$ & $1.3 \mathrm{pF}$ & $14 \mathrm{ps}$ \\
\hline $\mathbf{1} \mathbf{p F}$ & $2.3 \mathrm{pF}$ & $20 \mathrm{ps}$ \\
\hline $\mathbf{2} \mathbf{p F}$ & $3.3 \mathrm{pF}$ & $27 \mathrm{ps}$ \\
\hline $\mathbf{3} \mathbf{p F}$ & $4.3 \mathrm{pF}$ & $40 \mathrm{ps}$ \\
\hline
\end{tabular}

Table 2: jitter for various $C d$

The measured power consumption of the preamp and of the discriminator is respectively $440 \mu \mathrm{W}$ and $360 \mu \mathrm{W}$, which meets the requirement of a power consumption of less than 1 $\mathrm{mW} / \mathrm{channel}$.

Test beam measurements were performed at CERN mid-September using this test board and an ALTIROC0 ASIC bump-bonded to a four $1 \times 1 \mathrm{~mm}^{2}$ LGAD sensor. Data analysis is ongoing and first results are quite promising

\section{Conclusion}

Test bench measurements of ALTIROC0 show a jitter better than $30 \mathrm{ps} r m s$ for an actual detector capacitance of $3.4 \mathrm{pF}$ and an injected charge of $10 \mathrm{fC}$ corresponding to 1 MIP with an LGAD gain of 20. The power consumption of the analog part is $800 \mu \mathrm{W}$, which is smaller than the requirement of $1 \mathrm{~mW} /$ channel. A next version of the ASIC, ALTIROC1, with 16 or 25 complete readout channels, is foreseen. Each channel will integrate the front end followed by two 20 ps-bin Time-to-Digital-Converters for the TOT and TOA digitization and a memory to store the data.

\section{Acknowledgement}

This project has received funding from the European Union's Horizon 2020 Research and Innovation programme under Grant Agreement no. 654168.

\section{References}

[1] : ATLAS Phase-II Upgrade Scoping Document, CERN-LHCC-2015-020. LHCC-G-166, CERN, 2015, https://cds.cern.ch/record/2055248 